\title{
New extended Jacobi elliptic function expansion scheme for wave-wave interaction in ionic media
}

\author{
Ram Dayal Pankaj $^{1 *}$, Bhawani Singh ${ }^{1}$, Arun Kumar ${ }^{2}$ \\ ${ }^{1}$ Department of Mathematics, J. N. V. University, Jodhpur, India \\ ${ }^{2}$ Department of Mathematics, Government College Kota, Kota (Raj.), India \\ *drrdpankaj@yahoo.com
}

DOI 10.17586/2220-8054-2018-9-5-581-585

\begin{abstract}
New Jacobi Elliptic functions expansion scheme, more general than the hyperbolic tangent function method, is derived to construct the exact wave solutions in terms of Jacobi Elliptic functions. The coupled 1D nonlinear Schrödinger-Zakharov (CNLSZ) system is taken as the model equation for wave-wave interaction in ionic media. It is shown that more new solutions can be obtained at their limit condition.
\end{abstract}

Keywords: the coupled 1D nonlinear Schrödinger-Zakharov (CNLSZ) system, Jacobi elliptic function expansion scheme, hyperbolic tangent function expansion.

Received: 16 May 2018

Revised: 6 August 2018

\section{Introduction}

It is well known that nonlinear physical phenomena are related to nonlinear partial differential equations, which are employed in natural and applied science fields such as fluid dynamics, plasma physics, biology, etc. One of the most fundamental and charming phenomena in plasma physics is Langmuir turbulence [1,2]. Although, in the lowamplitude linear limit, this turbulence consists only of high-frequency electron oscillations, the presence of larger amplitude waves induces nonlinearities which couple the high-frequency electron oscillations to low-frequency ion oscillations. These nonlinearities lead to parametric instabilities, including a three-wave interaction called the parametric decay instability and a four-wave interaction called the modulational instability or oscillating two stream instability [3]. The strongly nonlinear state leads to the formation of coherent structures called solitons [4]; these structures are stable in one dimension and can collapse catastrophically in two or three dimensions [5-9]. Zakharov [5] introduced a relatively simple set of fluid equations to describe all of these physical phenomena. In the single spatial dimension, the Zakharov equations are:

$$
\begin{aligned}
i E_{t}+E_{x x}-\eta E & =0, \\
\eta_{t t}-\eta_{x x}-|E|_{x x}^{2} & =0,
\end{aligned}
$$

where $t$ is dimensionless time, $x$ is dimensionless distance, $E(x, t)$ is the dimensionless slowly varying envelope of the high-frequency electric field, and $\eta(x, t)$ is the dimensionless low-frequency density variation. The numerical and analytic study of the properties of equation (1) is a very active area of research in fundamental plasma physics [5-13]. Much effort has been spent on the construction of exact solutions of nonlinear equations for their important role in understanding the nonlinear problems. Physically, the wave-wave interaction or the wave collisions are common phenomena in science and engineering for both solitary and non-solitary waves. At the classical level, a set of coupled nonlinear wave equations describing the interaction between high-frequency Langmuir waves and low-frequency ion-acoustic waves were firstly derived by Zakharov [5]. Since then, this system has been the subject of a large number of studies. Currently, there are many methods of constructing exact solutions, for instance, the inverse scattering transform [14], the Hirota method [15], the Backlund method [16], the extended tanh-function method [17], the variational method [18], the Adomian methods decomposition method [1922] and several other numerical [23-27]. In this article, we will present some new solutions of Jacobi elliptic function type of CNLSZ equation by using an extended Jacobi elliptic function method. 


\section{The extended Jacobi elliptic function expansion method}

We now present briefly the main steps of the extended Jacobi elliptic function expansion strategy that will be applied. Consider a given (second order and cubic nonlinear) nonlinear wave equation

$$
P\left(u, u_{t}, u_{x}, u_{t t}, u_{x x}, \ldots\right)=0,
$$

can be converted to an ODE

$$
P\left(u, u^{\prime}, u^{\prime \prime}, u^{\prime \prime \prime}, \ldots\right)=0,
$$

upon using a wave variable $\xi=\alpha(x-c t)$, where $\alpha$ and $c$ are the wave number and wave speed, respectively equation (3) is then integrated as long as all terms contain derivatives where integration constants are considered zeros. Introducing a new independent variable $Y=Y(\xi)$, by the Jacobi elliptic function expansion method, $Y(\xi)$ can be expressed as a finite series of Jacobi elliptic functions,

$$
Y(\xi)=\sum_{i=0}^{n} a_{i}(\operatorname{sn}(\xi)+\lambda d n(\xi))^{i}
$$

is made and $a_{i}(i=1,2,3, \ldots n)$ and $\lambda \neq 0$ are constant. Its highest degree is $O\{Y(\xi)\}=n, n$ is positive number can be determined by consisting the homogeneous balance the highest order derivative and highest nonlinear appearing the ODE.

$s n(\xi)=s n(\xi, m), d n(\xi)=d n(\xi, m)$ and $c n(\xi)=c n(\xi, m)$ are the Jacobi elliptic function with modulus $m$, where $0<m<1$. These functions satisfy the following formulas:

$s n^{2}(\xi)+c n^{2}(\xi)=1, \quad d n^{2}(\xi)+m^{2} s n^{2}(\xi)=1 \quad$ and $\quad s n^{\prime}(\xi)=c n(\xi) d n(\xi), \quad d n^{\prime}(\xi)=-m^{2} c n(\xi) s n(\xi)$.

These functions degenerate into hyperbolic functions when $m>1$ as follows:

$$
s n(\xi) \rightarrow \tanh (\xi), \quad c n(\xi) \rightarrow \sec h(\xi), \quad d n(\xi) \rightarrow \sec h(\xi) .
$$

We can select $n$ in (4) to balance the derivative term of the highest order and the nonlinear term. So, the Jacobi elliptic function expansion method is more general than the hyperbolic tangent function expansion method.

\section{Application extended Jacobi elliptic function expansion method for CNLSZ system}

We introduce a transformation for (GZE) equation (1):

$$
\begin{gathered}
E(x, t)=U(\xi) e^{i \theta}, \quad \eta(x, t)=V(\xi), \\
\theta=k x-\omega t, \quad \xi=p(x-2 k t),
\end{gathered}
$$

where $k, \omega$ and $p$ are real constant. Put these transformation in equation (1), we have the ordinary differential equation (ODE) for $U(\xi)$ and $V(\xi)$ :

$$
\begin{gathered}
p^{2} U^{\prime \prime}(\xi)-U(\xi)\left(k^{2}-\omega\right)-V(\xi) U(\xi)=0, \\
\left(4 k^{2}-1\right) V^{\prime \prime}(\xi)+U^{\prime \prime}(\xi)=0,
\end{gathered}
$$

where prime denotes the differential with respect to $\xi$. Integration of second equation of system (5) twice with respect to $\xi$ affords:

$$
V(\xi)=\frac{C_{1}-U^{2}(\xi)}{\left(4 k^{2}-1\right)}
$$

where $C$ is second integration constant and the first one is taken to zero. The value of $V(\xi)$ is put in first equation (5):

$$
\begin{gathered}
p^{2} U^{\prime \prime}(\xi)-U(\xi)\left(k^{2}-\omega+\frac{C_{1}}{4 k^{2}-1}\right)+\frac{U^{3}(\xi)}{4 k^{2}-1}=0 \\
A U^{\prime \prime}(\xi)-\beta U(\xi)+c U^{3}(\xi)=0
\end{gathered}
$$

where $\beta=\left(k^{2}-\omega+\frac{C_{1}}{4 k^{2}-1}\right), c=\frac{1}{4 k^{2}-1}, A=p^{2}$ is obtained after integrating the ODE once and setting the constant of integration equal to zero. Balancing $U^{\prime \prime}$ with $U^{3}$ in equation (7) gives $m+2=3 m$ i.e. $m=1$.

The new Jacobi elliptic function expansion method (5) admits the use of the finite expansion:

$$
U(\xi)=a_{0}+a_{1} \operatorname{sn}(\xi)+a_{1} \lambda d n(\xi) .
$$


Substituting equation (8) into equation (7) and:

$$
\begin{gathered}
A\left[-\left(1+m^{2}\right) a_{1} s n(\xi)+2 m^{2} s n^{3}(\xi)+\left(2-m^{2}\right) a_{1} d n(\xi)-2 a_{1} \lambda d n^{3}(\xi)\right]-\beta\left[a_{0}+a_{1} s n(\xi)+a_{1} \lambda d n(\xi)\right]+ \\
c\left[a_{0}^{3}+a_{1}^{3} s n^{3}(\xi)+a_{1}^{3} \lambda^{3} d n^{3}(\xi)+3 a_{1}^{3} \lambda s n^{2}(\xi) d n(\xi)+3 a_{1}^{3} \lambda^{2} \operatorname{sn}(\xi) d n^{2}(\xi)+3 a_{0}^{2} a_{1} \operatorname{sn}(\xi)+\right. \\
\left.3 \lambda a_{0}^{2} a_{1} \lambda d n(\xi)+3 a_{1}^{2} a_{0} s n^{2}(\xi)+6 a_{1}^{2} a_{0} \lambda s n(\xi) d n(\xi)+3 a_{1}^{2} a_{0} \lambda d n^{2}(\xi)\right]=0
\end{gathered}
$$

equating all coefficients with the powers in $s n(\xi), d n(\xi)$ and constant setting each of the obtained coefficients for functions to zero, yields the set of algebraic equations for $a_{0}, a_{1}, p, k, \omega$ and $\lambda$,

$$
\begin{aligned}
-\beta a_{0}+c a_{1}^{3} & =0 \\
-\left(1+m^{2}\right) A a_{1}-\beta a_{1}+c\left(3 a_{0}^{2} a_{1}\right) & =0 \\
A\left(\left(2-m^{2}\right) a_{1}\right)-\beta a_{1} \lambda+3 c \lambda a_{0}^{2} a_{1} & =0 \\
2 A m^{2} a_{1} \lambda+c a_{1}^{3} & =0 \\
-2 A a_{1} \lambda+c a_{1}^{3} \lambda^{3} & =0
\end{aligned}
$$

we obtain the sets of solutions.

Solutions - 1:

Thus:

$$
\lambda^{2}=a_{1}, \quad a_{0}^{2}=\frac{\left(\beta-A\left(2-m^{2}\right)\right)}{3 c}, \quad a_{1}^{2}=\frac{2 \beta+A\left(2-m^{2}\right)}{9 c} .
$$

$$
U(\xi)=\sqrt{\frac{\left(\beta-A\left(2-m^{2}\right)\right)}{3 c}}+\sqrt{\frac{2 \beta+A\left(2-m^{2}\right)}{9 c}}\{s n(p(x-2 k t))+\lambda d n(p(x-2 k t))\} .
$$

If $m \rightarrow 1$ then equation (9):

$$
U(\xi)=\sqrt{\frac{(\beta-A)}{3 c}}+\sqrt{\frac{2 \beta+A}{9 c}}\{\tanh (p(x-2 k t))+\lambda \operatorname{sech}(p(x-2 k t))\} .
$$

If $m \rightarrow 0$ then equation (9):

Solutions - 2:

$$
U(\xi)=\sqrt{\frac{(\beta-A)}{3 c}}+\sqrt{\frac{2 \beta+2 A}{9 c}}\{\tan (p(x-2 k t))+\lambda \sec (p(x-2 k t))\} .
$$

Thus:

$$
\lambda^{2}=a_{1}, \quad a_{0}^{2}=\frac{\left(\beta-A\left(2-m^{2}\right)\right)}{3 c}, \quad a_{1}^{2}=\frac{1+m^{2}}{m^{2}-2} .
$$

$$
U(\xi)=\sqrt{\frac{\left(\beta-A\left(2-m^{2}\right)\right)}{3 c}}+\sqrt{\frac{1+m^{2}}{m^{2}-2}}\{\operatorname{sn}(p(x-2 k t))+\lambda d n(p(x-2 k t))\} .
$$

If $m \rightarrow 1$ then equation (12):

$$
U(\xi)=\sqrt{\frac{(\beta-A)}{3 c}}+\sqrt{-2}\{\tanh (p(x-2 k t))+\lambda \operatorname{sech}(p(x-2 k t))\} .
$$

If $m \rightarrow 0$ then equation (12):

$$
U(\xi)=\sqrt{\frac{(\beta-2 A)}{3 c}}+\sqrt{-\frac{1}{2}}\{\tan (p(x-2 k t))+\lambda \sec (p(x-2 k t))\} .
$$

Solutions - 3:

$$
\begin{gathered}
\lambda= \pm m, \quad a_{1}^{2}=\frac{2 A}{m^{2} c}, \quad a_{0}^{2}=1-\frac{6 A}{\beta m^{2}} . \\
U(\xi)=\sqrt{1-\frac{6 A}{\beta m^{2}}}+\sqrt{\frac{2 A}{m^{2} c}}\{\operatorname{sn}(p(x-2 k t)) \pm m d n(p(x-2 k t))\} .
\end{gathered}
$$

If $m \rightarrow 1$ then equation (15):

$$
U(\xi)=\sqrt{1-\frac{6 A}{\beta}}+\sqrt{\frac{2 A}{c}}\{\tanh (p(x-2 k t)) \pm \operatorname{sech}(p(x-2 k t))\} .
$$

Solutions - 4:

$$
a_{1}^{2}=\frac{A\left(m^{2}-2\right)-2 \beta}{3 c}, \quad a_{0}^{2}=\frac{\beta}{c}, \quad \lambda^{2}=\frac{6 A+3 m^{2}\left[A\left(m^{2}-2\right)-2 \beta\right]}{\left(A\left(m^{2}-2\right)-2 \beta\right)},
$$




$$
U(\xi)=\sqrt{\frac{\beta}{c}}+\sqrt{\frac{A\left(m^{2}-2\right)-2 \beta}{3 c}}\left\{s n(p(x-2 k t))+\sqrt{\frac{6 A+3 m^{2}\left[A\left(m^{2}-2\right)-2 \beta\right]}{A\left(m^{2}-2\right)-2 \beta}} d n(p(x-2 k t))\right\} .
$$

If $m \rightarrow 1$ then equation (17):

$$
U(\xi)=\sqrt{\frac{\beta}{c}}+\sqrt{-\frac{A+2 \beta}{3 c}}\left\{\tanh (p(x-2 k t))+\sqrt{\frac{2 \beta-3 A}{A+2 \beta}} \sec h(p(x-2 k t))\right\} .
$$

If $m \rightarrow 0$ then equation (17):

$$
U(\xi)=\sqrt{\frac{\beta}{c}}+\sqrt{-2 \frac{(A+\beta)}{3 c}}\left\{\tan (p(x-2 k t))+\sqrt{\frac{-3 A}{A+\beta}} \sec (p(x-2 k t))\right\} .
$$

\section{Conclusion}

The Jacobi elliptic function expansion method is proposed and applied to the coupled 1D nonlinear SchrödingerZakharov (CNLSZ) system. Using this method, we found some new solutions for the Jacobi elliptic function type that were not obtained by the sine-cosine method, the extended tanh-method, the mapping method, and other methods. In the limiting case of the Jacobi elliptic function (namely, modulus setting 0 or 1 ), we also obtained the solutions of sin-type, cos-type, tanh-type, sech-type. In the applications, the solutions are completely new and have not found in earlier. But the solutions found in the Ref. [27] are the same to our obtain solutions -3 . By means of this scheme, we found some new solutions of the above mentioned equations. Therefore, the scheme can be easily applied to solve the NLDEs and provides some new solutions. The solutions obtained in this article have been verified by plugging them back into the original equation and found correct. It is shown that this scheme can be applied to more coupled equations. So many new shock wave or solitary wave solutions can also be obtained. Actually, this method can be applied to obtain solutions and classify modulational instability to more nonlinear wave equations, as long as the odd- and even-order derivative terms do not coexist in the nonlinear wave equations.

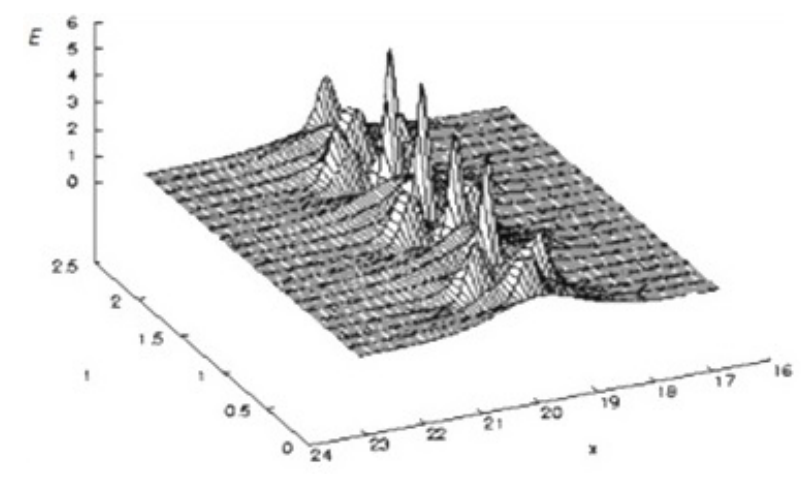

FIG. 1. Exact solitary wave solution of $E(x, t)$ with fixed value of $k=1 / 2$ for different values of time

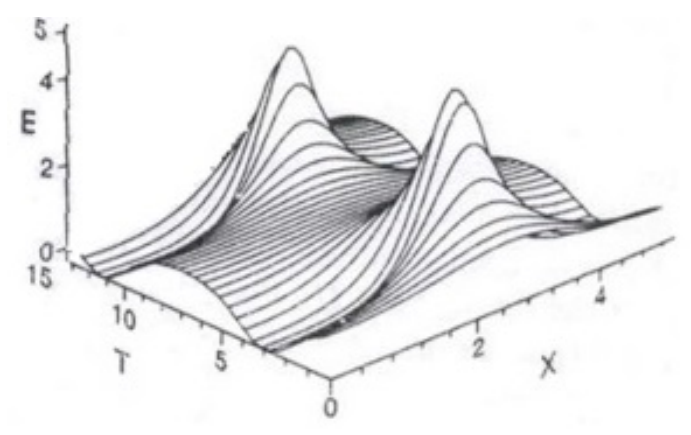

FIG. 2. Time evolution of the Nonlinear equation by the variational approach for $k=1$ 


\section{References}

[1] Langmuir I. Oscillations in Ionized Gases. Proc. Natl. Acad. Sci. U.S.A., 1928, 14 (8), P. 627-637.

[2] Tonks L., Langmuir I. Oscillations in Ionized Gases. Physical Review, 1929, 33, P. 195-210.

[3] Chen F.F. Introduction to Plasma Physics, Plenum, New York, 1974.

[4] Scott C., Chu F.Y.F., Mcglaughlin D.W. The soliton: a new concept in applied science. Proc. IEEE, 1973, 61, P. 1443-1483.

[5] Zakharov V.E. Collapse of Langmuir Waves. JEPT, 1972, 35 (5), P. 908-914.

[6] Pereira N.R., Sudan R.N., Denavit J. "Numerical study of two-dimensional generation and collapse of Langmuir solitons. The Physics of Fluids, 1977, 20 (6), P. 936-945.

[7] Nicholson D.R., et al. Nonlinear Langmuir waves during type III solar radio bursts. The Astrophysical Journal, 1978,223 , P. 605-619.

[8] Goldman M.V., Nicholson D.R. Virial theory of direct Langmuir collapse. Physical Review Letters, 1978,41 (6), P. 406.

[9] Nicholson R., Goldman M.V. Cascade and collapse of Langmuir waves in two dimensions. Phys. Fluids, 1978,21 (10), P. $1766-1776$.

[10] Hasegawa A. Stimulated Modulational Instabilities of Plasma Waves. Phys. Rev. A, 1970, 1 (6), P. 1746.

[11] Thyagaraja A. Recurrence, dimensionality, and Lagrange stability of solutions of the nonlinear Schrödinger equation. Phys. of Fluids, 1981, 24 (11), P. 1973-1975.

[12] Russell D.A., Ott E. Chaotic (strange) and periodic behavior in instability saturation by the oscillating twostream instability. The Physics of Fluids, 1981, 24 (11), P. 1976-1988.

[13] Weatherall J.C., et al. Solitons and ionospheric heating. Journal of Geophysical Research: Space Physics, 1982,87 (A2), P. $823-832$.

[14] Vakhnenko V.O., Parkes E.J., Morrison A.J. A Backlund transformation and the inverse scattering transform method for the generalised Vakhnenko equation. Chaos, Solitons \& Fractals, 2003, 17 (4), P. 683-692.

[15] Ye-peng Sun, Jin-bo Bi, Deng-yuan Chen. N-soliton solutions and double Wronskian solution of the non-isospectral AKNS equation. Chaos, Solitons \& Fractals, 2005, 26 (3), P. 905-912.

[16] Sakka A. Backland transformations for Painleve I and II equations to Painleve-type equations of second order and higher degree. Phys. Lett. A, 2002, 300 (2-3), P. 228-232.

[17] Yao R.X., Li Z.B. New exact solutions for three nonlinear evolution equations. Phys. Lett. A, 2002, 297, P. $196-204$.

[18] Kumar A. An analytical solution for a coupled partial differential equation. Applied Mathematics and Computation, 2009, 212, P. 245-250.

[19] Liu Z., Chen C. Compactons in a general compressible hyper elastic rod. Chaos, Solitons \& Fractals, 2004,22 (3), P. 627-640.

[20] Kaya D., El-Sayed S.M. An application of the decomposition method for the generalized KdV and RLW equations. Chaos, Solitons \& Fractals, 2003, 17 (5), P. 869-877.

[21] Hagtae Kim, Dong Pyo Hong, Kil to Chong. A numerical solution of point kinetics equations using the Adomian Decomposition Method. Systems and Informatics (ICSAI), 2012 International Conference on IEEE 19-20 May 2012, 12835470.

[22] Kumar A., Ram D.P. Solitary Wave Solutions of Schrödinger Equation by Laplace-Adomian Decomposition Method. Physical Review \& Research International, 2013, 3 (4), P. 702-712.

[23] Ghoreishi M., Ismail A.I.B., Rashid A. Numerical Solution of Klein-Gordon-Zakharov Equations using Chebyshev Cardinal Functions. Journal of Computational Analysis \& Applications, 2012, 14 (1), P. 574-582.

[24] Zhao Xiaofei, Ziyi Li. Numerical Methods and Simulations for the Dynamics of One-Dimensional Zakharov-Rubenchik Equations. Journal of Scientific Computing, 2013, 59 (2), P. 412-438.

[25] Kumar A., Ram D.P., Manish G. Finite difference scheme of a model for nonlinear wave-wave interaction in ionic media. Computational Mathematics and Modeling, 2011, 22 (3), P. 255-265.

[26] Kumar A., Ram D.P. Finite Difference Scheme for the Zakharov Equation as a Model for Nonlinear Wave-Wave Interaction in Ionic Media. International Journal of Scientific \& Engineering Research, 2014, 5 (2), P. 759-762

[27] Kumar A., Ram D.P. Solitary Wave Solutions of Schrödinger Equation by Laplace-Adomian Decomposition Method. Physical Review \& Research International, 2013, 3 (4), P. 702-712. 